interesting and difficult biological problems across scales.

\author{
Kutti R. Vinothkumar (iD $ه$ \\ National Centre for Biological Sciences TIFR, \\ Bangalore, India. \\ $\bigotimes_{e-m a i l: v k u m a r @ n c b s . r e s . i n}$
}

Published online: 7 May 2021

https://doi.org/10.1038/s41592-021-01139-x

\section{References}

1. Su, C. C. et al. Nat. Methods 18, 69-75 (2021).

2. Naydenova, K., Jia, P. \& Russo, C. J. Science 370, 223-226 (2020).

3. O'Reilly, F. J. et al. Science 369, 554-557 (2020).

4. Subramanian, R., Mayor, S. \& Vinothkumar, K. R. Biophys. Rev. 11, 513-514 (2019)
Acknowledgements

The author acknowledges funding from Department of Biotechnology, India, B-Life grant DBT/PR12422/ MED/31/287/2014; the Department of Atomic Energy, Government of India, under Project Identification No. RTI4006; and SERB, India, for the Ramanujan Fellowship (RJN-094/2017).

Competing interests

The author declares no competing interests.

\title{
RNA structure: a renaissance begins?
}

Check for updates

\section{Advances in cryo-EM technology will open a new era of RNA-only 3D structure determination.}

\author{
Rhiju Das
}

W here are all the RNA structures? For decades, RNA has been known to act as both a genetic script and a molecular machine, and the amount of RNA sequence transcribed in organisms like humans exceeds the number of protein sequences by at least tenfold ${ }^{1}$. Numerous RNA structures have been deposited in the Protein Data Bank (PDB), which, despite its name, remains the biology community's storehouse of structural information for both RNA and proteins. But a quick search in the PDB reveals a serious imbalance tipped in favor of protein structures: the fraction of RNA-only structures remains below $1 \%$. Biologists-even RNA structural biologists-would be forgiven for assuming that most RNAs don't fold. Or, if RNAs do fold, their structures might be ill-defined in three dimensions unless organized by partners like proteins. As a molecule, RNA has been called 'floppy', 'flexible' and 'conformationally heterogeneous', and this reputation has scared off all but the boldest from attempting crystallography or NMR on RNA-only structures. Is determining RNA three-dimensional (3D) structure a lost cause?

A couple of years ago, the field began to revisit the determination of RNA-only structures with cryogenic electron microscopy (cryo-EM), not expecting much but excited by the technique's clear power in imaging ribosomes, spliceosomes and other RNAs complexed to proteins. To everyone's surprise, protein-free, RNA-only complexes in fact did lend themselves to imaging by cryo-EM. Newly visualizable molecules included the Tetrahymena ribozyme, the first RNA-only enzyme discovered in nature (Fig. 1a) ${ }^{2}$; its global structure had remained intractable for four decades. Other RNAs studied using cryo-EM were smaller than $30 \mathrm{kDa}$, well below the $\sim 100$ $\mathrm{kDa}$ detection limit expected for proteins ${ }^{3}$, and maps with sub-4-Å resolution even allowed detection of small-molecule ligands ${ }^{4}$. In 2020, the COVID-19 pandemic prompted

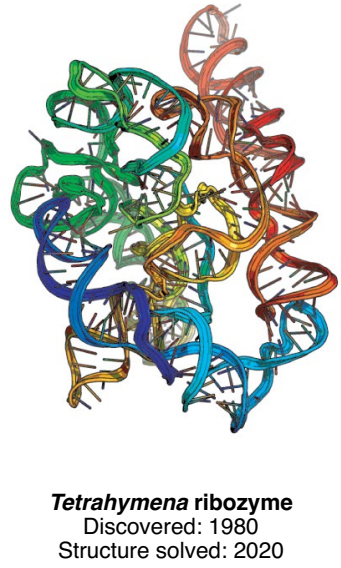

b

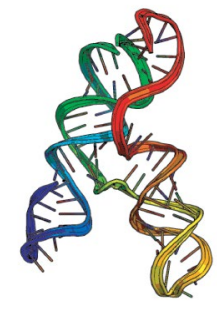

SARS-CoV-2 frameshift element Discovered: 2020 Structure solved: 2020

Fig. 1 | Coordinates of RNA molecules with complex folds revealed through cryogenic electron microscopy. a, Tetrahymena ribozyme, first RNA-only enzyme discovered (PDB 6WLS). b, SARS-CoV-2 frameshift stimulation element, a highly conserved potential target for COVID-19 antivirals that reprograms the ribosome (PDB 6XRZ).

testing of even smaller RNAs, including a 28 $\mathrm{kDa}$ frameshift stimulation element from the SARS-CoV-2 genome-and cryo-EM delivered again (Fig. 1b) ${ }^{5}$. Each of these structures revealed homologies to other RNAs; some structures revealed holes and pockets that might be targeted by drugs for potential biomedical disruption. For RNAs, as for proteins, structure truly can illuminate function.

These results suggest that RNA 3D structures are there for the finding. Indeed, RNA may be the perfect molecule for cryo-EM, and cryo-EM may be the perfect technique for RNA. At the time of writing, however, it is still early days for RNA-only cryo-EM. How far can the resolution of cryo-EM be pushed for RNA-only systems, especially if they form multiple biologically important states? How many RNA domains with well-defined, biologically relevant $3 \mathrm{D}$ structures are there really in the human transcriptome-or the Earth's transcriptome?
If the answers are favorable, an RNA structure renaissance has begun, and in another 50 years our 3D structural database may very well be dominated by RNAs, not proteins. Perhaps the Protein Data Bank will need another name. $\square$

\section{Rhiju Das (1D)}

Departments of Biochemistry and Physics, Stanford University, Stanford, CA, USA.

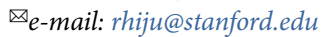

Published online: 7 May 2021

https://doi.org/10.1038/s41592-021-01132-4

References

1. Mattick, J. Nat. Rev. Genet. 5, 316-323 (2004).

2. Kappel, K. et al. Nat. Methods 17, 699-707 (2020).

3. Henderson, R. Q. Rev. Biophys. 28, 171-193 (1995).

4. Zhang, K. et al. Nat. Commun. 10, 5511 (2019).

5. Zhang K. et al. Preprint at bioRxiv https://doi. org/10.1101/2020.07.18.209270 (2020).

The author declares no competing interests. 